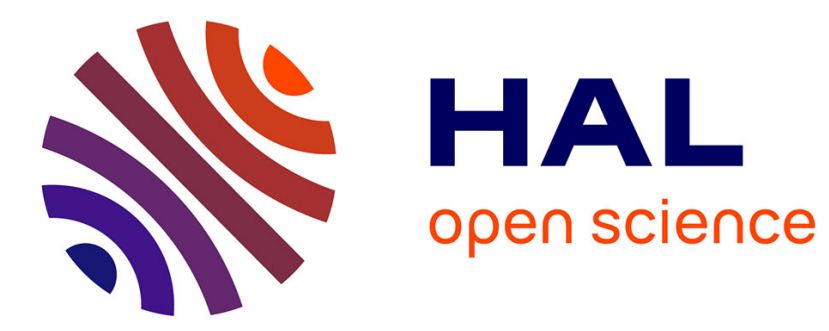

\title{
The Relationship between Anthropometric Variables and Features of Electromyography Signal for Human-Computer Interface
}

\author{
Angkoon Phinyomark, Franck Quaine, Yann Laurillau
}

\section{To cite this version:}

Angkoon Phinyomark, Franck Quaine, Yann Laurillau. The Relationship between Anthropometric Variables and Features of Electromyography Signal for Human-Computer Interface. Dr. Ganesh Naik. Applications, Challenges, and Advancements in Electromyography Signal Processing, IGI Global, 2014, 9781466660908. 10.4018/978-1-4666-6090-8 . hal-01006862

\section{HAL Id: hal-01006862 \\ https://inria.hal.science/hal-01006862}

Submitted on 16 Jun 2014

HAL is a multi-disciplinary open access archive for the deposit and dissemination of scientific research documents, whether they are published or not. The documents may come from teaching and research institutions in France or abroad, or from public or private research centers.
L'archive ouverte pluridisciplinaire HAL, est destinée au dépôt et à la diffusion de documents scientifiques de niveau recherche, publiés ou non, émanant des établissements d'enseignement et de recherche français ou étrangers, des laboratoires publics ou privés. 


\title{
The Relationship between Anthropometric Variables and Features of Electromyography Signal for Human-Computer Interface
}

\author{
Angkoon Phinyomark ${ }^{1,2}$, Franck Quaine ${ }^{1}$, Yann Laurillau ${ }^{2}$ \\ ${ }^{1}$ GIPSA Laboratory, CNRS UMR 5216, Department of Control System, SAIGA Team, \\ University Joseph Fourier, Grenoble, France \\ ${ }^{2}$ LIG Laboratory, CNRS UMR 5217, University of Grenoble, Grenoble, France
}

\begin{abstract}
Muscle-computer interfaces (MCIs) based on surface electromyography (EMG) pattern recognition have been developed based on two consecutive components: feature extraction and classification algorithms. Many features and classifiers are proposed and evaluated, which yield the high classification accuracy and the high number of discriminated motions under a single-session experimental condition. However, there are many limitations to use MCIs in the real-world contexts, such as the robustness over time, noise, or low-level EMG activities. Although the selection of the suitable robust features can solve such problems, EMG pattern recognition has to design and train for a particular individual user to reach high accuracy. Due to different body compositions across users, a feasibility to use anthropometric variables to calibrate EMG recognition system automatically/semi-automatically is proposed. This chapter presents the relationships between robust features extracted from actions associated with surface EMG signals and twelve related anthropometric variables. The strong and significant associations presented in this chapter could benefit a further design of the MCIs based on EMG pattern recognition.
\end{abstract}

\section{INTRODUCTION}

Surface electromyography (EMG) signals are measured by surface electrodes that are placed on the target muscles. During muscle contractions, a compound of the whole motor unit action potentials (MUAPs) occurred in the muscles. These MUAPs are the useful information for numerous fields, e.g. rehabilitation engineering, biomechanics, ergonomics, and human-computer interfaces (HCIs) (Merletti \& Parker, 2004). Surface EMG signals can also be used in a medical decision support system, e.g. the diagnosis of neuromuscular disorders (Subasi, 2012, 2013). In this chapter, we focus on the development of the HCIs based on surface EMG signals, as called "muscle-computer interfaces or MCIs" (Saponas, Tan, Morris, \& Balakrishnan, 2008). These interfaces can widely use in controlling many external devices, e.g. prosthetic limbs, electric-power wheelchairs, interactive surfaces, a virtual computer mouse or keyboard, a portable music player, and in-car electronic equipment (e.g. Barreto, Scargle, \& Adjouadi, 2000; Benko, Saponas, Morris, \& Tan, 2009; Khushaba, Kodagoda, Liu, \& Dissanayake, 2013; Saponas et al., 2009; Shenoy, Miller, Crawford, \& Rao, 2008; Wei, Hu, \& Zhang, 2011; Yang, Lin, Lin, \& Lee, in press). 
MCIs generally consist of three main modules, as shown in Fig. 1. The first module composes of two submodules: surface EMG signal acquisition (hardware) and data pre-processing (software). In the data acquisition sub-module, surface EMG signals are firstly amplified with an amplifier due to small EMG amplitude, and may be filtered by hardware filters, i.e., notch, high pass and/or low pass filters. Then, continuous surface EMG signals are sampled using an analog-to-digital converter. In case of no hardware filters, the raw EMG data can be filtered by software filters, before the EMG data go to the next module, the pattern recognition module. Different patterns of surface EMG signals are classified and matched to the control commands in this module. The second module can be divided into three sub-modules: feature extraction, dimensionality reduction, and classification algorithms. All sub-modules are in the software part. The pre-processed surface EMG data from the first module are segmented into small time slot length using an adjacent or an overlapped windowing technique, and then some features are extracted in order to emphasize the relevant structures in the EMG signals and remove noises/irrelevant parts. A feature vector is formed and can be sent directly to a classifier, or the dimension of a feature vector is reduced by the dimensionality reduction technique before sending it to the classifier. The classifier maps the extracted features (the representative of the actions associated with surface EMG signals) to the target classes (the control commands for an external device). After the control commands were generated based on the mathematical functions in the second module, the third module is a control system, which serves as an interface between the software and hardware. In other words, an output command is converted from a digital code to an analog signal for controlling an external device. In this chapter, we focus on the second module, particularly the extraction of EMG features.

\section{Figure 1. Three main modules of MCIs: the surface EMG acquisition and pre-processing, the pattern recognition, and the control system.}

Nearly all previous studies on MCIs based on EMG pattern recognition concentrate on improving recognition rate together with increasing the number of discriminated motions. Currently, the recognition rate is more than $90 \%$ in discriminating 4-12 finger, wrist, hand, and forearm motions. It should be noted that the recognition rate or the classification accuracy is calculated as a ratio of the number of correct classifications to the total number of classifications. More details about many previous studies on MCIs based on EMG pattern recognition concentrate on improving recognition rate can be found in additional readings in the Additional Reading Section. However, the high recognition rates reported are usually based in single-session experiments conducted in research laboratories (Tkach, Huang, \& Kuiken, 2010). On the other hand, in clinics when the context of the real-world requirements have been given attention, there are many issues that have to find the solutions, e.g. the robustness against a variety of noises (Boostani \& Moradi, 2003; Zardoshti-Kermani, Wheeler, Badie, \& Hashemi, 1995), the robustness over time-between sessions (Oskouei, Paulin, \& Carman, 2013; Sensinger, Lock, \& Kuiken, 2009) and between days (Boschmann, Kaufmann, Platzner, \& Winkler, 2010; Zhang et al., 2008)-due to EMG electrode location shift (Young, Hargrove, \& Kuiken, 2011, 2012), muscle fatigue, and/or variation in muscle contraction effort (Tkach et al., 2010), or the robustness in limb position variations (Chen, Geng, \& Li, 2011; Fougner et al., 2011; Jiang, Muceli, Graimann, \& Farina, 2013).

Among such issues, the problem of the cross-user classification is far from being a practical one (Cannan $\& \mathrm{Hu}, 2011$; Saponas et al., 2008). The proposed systems in the literature were trained and tested independently on the EMG signals from each user. In other words, EMG-based MCIs have to design and create for each user. Based on a preliminary study of Saponas et al. (2008), when the classifier is trained on the data from all subjects in the database except the testing subject, the classification accuracy computed from the testing subject data is about 50\%, reducing from about $95 \%$ using the independent training and testing system. It should be noted that their system classifies five single finger motions based on three time-domain and frequency-domain features extracted from eight EMG channels placed around the upper forearm (Saponas et al., 2008). Moreover, based on our preliminary experiments, the selection 
of the robust EMG features is not an efficient solution to reduce the variability of surface EMG signals between users, like other previous mentioned problems.

This is due to the different compositions of the body across users, which result in the different muscle types with varying architecture characteristics. This factor prevents the development of standard MCIs that are compatible with almost any user, and MCIs based on EMG pattern recognition have never really reached the general population. Being able to solve this problem, in our previous work (Phinyomark et al., 2013a) a feasibility to use anthropometric variables, a measurable characteristic of the human body, to calibrate the EMG pattern recognition systems is presented. The anthropometric variables are used to compensate for discrepancies between user body compositions (Cannan \& Hu, 2011), which are based on the relationship between the EMG signal, muscle force, and muscle size consisting of cross-sectional area and length (Hof, Pronk, \& van Best, 1987; Marras \& Sommerich, 1991; see our preliminary workPhinyomark et al., 2013a-for more details about this relationship). They can automatically or semiautomatically calibrate a system. As a result of the proposed technique, EMG-based MCIs that require little or no user-specific training EMG data are possible. It is important to note that it is easy to measure anthropometric variables, and some variables can be measured directly together with surface EMG signals via an armband device (Cannan \& Hu, 2011; Saponas et al., 2008). It is also possible to obtain estimated anthropometric variables from the anthropometric table, which can be found in many sources including academic books (e.g. De Leva, 1996; Winter, 1990). It means that there is no need to measure all anthropometric variables and may need only a few basic variables, i.e., gender, age, body mass, or standing height. However, at the beginning all anthropometric variables proposed in this chapter are measured directly from the volunteers.

For simple MCI systems that use a thresholding technique, a maximum voluntary contraction (MVC) can be used as an additional input to calibrate and adapt the classification system from one user to another user. In order to use MVC normalization technique, it is important that the linear relationship between force generated and EMG level should be held for the muscle of interest up to the maximum force/EMG level (Bolgla \& Uhl, 2007; Vera-Garcia, Moreside, \& McGill, 2010). Although the linear relationship has been found in many previous works (e.g. Kamavuako, Farina, Yoshida, \& Jensen, 2009; Woods \& Bigland-Ritchie, 1983), several researchers have found a non-linear relationship between force generated and EMG muscle activity (e.g. Lawrence \& De Luca, 1983; Woods \& Bigland-Ritchie, 1983). The relationship is more complicated in case of a dynamic muscle contraction, i.e., a muscle is free to change length and a joint is free to move (Oatis, 2008). Several anthropometric variables have been found to have a strong relationship with the MVC and other techniques of maximum force/EMG measurement, such as hand circumference has the strong correlation with maximal grip strength (MGS) and can be used to predict the MGS using a simple regression equation in the study of $\mathrm{Li}$, Hewson, Duchene, \& Hogrel (2010), or in the study of Marras \& David (2001), several linear regression equations developed from five of the fifteen anthropometric variables are used to predict the EMC (Expected Maximum Contraction) of trunk muscles.

However, for advanced MCI systems, features extracted from surface EMG signals are the important stage, and only MVC (related only with muscle force) is not enough to be used alone. Other additional useful information is needed, which should have strong relationships with many EMG features (extracted not only force level information but also other useful information, e.g. complexity and frequency). So some additional inputs should be used to calibrate the machine learning and pattern recognition instead of the simple thresholding technique. Forearm circumference is an anthropometric variable that has already been proposed to be used as a calibrating variable in an EMG recognition system in previous works (Anakwe, Huntley, \& Mceachan, 2007; Cannan \& Hu, 2011). However, the relationship between EMG and forearm circumference is not strong enough, so further anthropometric variables were evaluated their relationships with surface EMG features in this chapter. All relationships between EMG features and anthropometric variables that are strong and significant could benefit a further design of the EMG-based 
MCIs. It could semi-automatically/automatically adapt the setting of the EMG recognition systems to a wider population. It can be used in two different ways: 1) a normalized value for EMG features, and 2) a weighting factor for a classifier. Moreover, wearable and wireless EMG devices are already commercially available, e.g. MYO (see www.thalmic.com/myo/), and has been developed rapidly, thus using anthropometric variables to calibrate the EMG recognition system would become more practical and important in the near future.

\section{EMG FEATURE EXTRACTION Definition of Feature Extraction}

Feature extraction is a technique to transform raw EMG signals into a reduced representation set of features as called "a feature vector". A transformation is usually based on mathematical functions, which highlights relevant structures in surface EMG signals and rejects irrelevant parts. If the extracted features are carefully selected, the feature vector will contain effective and relevant information drawn from the whole set of raw EMG data, which can be used to represent the desired actions. Training an EMG classifier on raw EMG signal patterns requires high computational cost and their testing classification performances are very poor (Phinyomark, Phukpattaranont, \& Limsakul, 2012e). Therefore, the extraction of EMG features is very important step in EMG-based gesture recognition. It is noted in the literature that the success of the EMG pattern recognition system almost entirely depends on the choice of features (Kendall et al., 2012; Hudgins, Parker, \& Scott, 1993).

EMG feature extraction can be computed in three domains: 1) time domain, 2) frequency domain, and 3) time-frequency or time-scale domain (Boostani \& Moradi, 2003). Time domain features are widely used in EMG pattern recognition due to the low complexity and computational cost, which can be implemented and computed using a simple hardware processor, i.e., a mobile device or a microprocessor (Hudgins et al, 1993; Kundu, Mazumder, \& Bhaumik, 2011). Moreover, time domain features can be successfully used as a dimensionality reduction technique for time-frequency/ time-scale features, i.e., the coefficients of short-time Fourier transform, discrete wavelet transform and wavelet packet transform (Phinyomark, Nuidod, Phukpattaranont, \& Limsakul, 2012b). Hence, in this chapter, features in time domain are mainly focused, and time-frequency/time-scale features are not considered. A number of frequency domain features are considered to be used in developing the robust MCI systems and to provide additional information.

\section{Computation of EMG Feature Extraction}

A basic idea about the computation of EMG feature extraction is illustrated in Fig. 2. Following is a brief description of all robust EMG features, which were investigated and discussed in the present chapter. In each, $x_{i}$ is the $i_{t h}$ sample of surface EMG signal amplitude and $L$ is the length of the analysis window for computing the features.

Figure 2. An example of EMG feature extraction procedure in time domain.

Willison Amplitude (WAMP) is a number of times resulting from the difference between two consecutive EMG amplitudes in a time segment becomes more than a predefined threshold thr. In other words, WAMP is a number of times that the length of the EMG waveform in a time segment exceeds a predefined threshold $t h r$, which can be expressed as

$$
\text { WAMP }=\sum_{i=1}^{L-1}\left[f\left(\left|x_{i+1}-x_{i}\right|\right)\right] ; f(x)=\left\{\begin{array}{ll}
1, & \text { if } x \geq t h r \\
0, & \text { otherwise }
\end{array} .\right.
$$


Myopulse Percentage Rate (MYOP) is an average number of times that the absolute value of EMG amplitude, i.e., myopulse output, exceeds a predefined threshold $t h r$, and the calculation is defined as

$$
\text { MYOP }=\frac{1}{L} \sum_{i=1}^{L}\left[f\left(\left|x_{i}\right|\right)\right] ; \quad f(x)=\left\{\begin{array}{ll}
1, & \text { if } x \geq t h r \\
0, & \text { otherwise }
\end{array} .\right.
$$

Slope Sign Change (SSC) or the number of turns (NT) is a number of times that the slope of the EMG waveform changes sign. This method uses three consecutive EMG signal amplitudes to detect the EMG waveform slope, which is calculated as

$$
\mathrm{SSC}=\sum_{i=2}^{L-1}\left[f\left[\left(x_{i}-x_{i-1}\right) \times\left(x_{i}-x_{i+1}\right)\right]\right] ; f(x)= \begin{cases}1, & \text { if } x \geq t h r \\ 0, & \text { otherwise }\end{cases}
$$

Zero Crossing (ZC) is a number of times that the EMG waveform crosses the zero amplitude axes. Several works used this value without implementing threshold condition (Boostani \& Moradi, 2003; Zardoshti-Kermani et al., 1995). However, a predefined threshold thr should be included in the ZC computation to reduce the effect of noise (Hudgins et al., 1993). ZC can be defined as

$$
\mathrm{ZC}=\sum_{i=1}^{L-1}\left[\operatorname{sgn}\left(x_{i+1} \times x_{i}\right) \cap\left|x_{i+1}-x_{i}\right| \geq t h r\right] ; \quad \operatorname{sgn}(x)= \begin{cases}1, & \text { if } x<0 \\ 0, & \text { otherwise }\end{cases}
$$

A suitable predefined threshold to reduce noise is dependent on a system gain value and a background noise level. So, we can roughly estimate a predefined threshold if we know both values (Hudgins et al, 1993). For example, assuming a system gain of 1000 and a background noise of $4 \mu \mathrm{V}$ peak to peak, the threshold can be calculated to be $\pm 2 \mathrm{mV}$. It is normally chosen between $50 \mu \mathrm{V}$ and $100 \mathrm{mV}$ (Philipson \& Larsson, 1988). However, to obtain a high quality feature space, the suitable threshold could be defined based on taking some offline trial-and-error classifications.

Detrended Fluctuation Analysis (DFA) is a modified root mean square analysis of a random walk (Peng, Havlin, Stanley, \& Goldberger, 1995). A slope of the line relating the logarithm of the RMS fluctuation of the profiles $y_{k}\left(F_{n}\right)$ to the logarithm of the predefined box size $(n)$ is used as a feature value $(\alpha)$. This value indicates the presence of the power law (fractal) scaling, as can be expressed as

$$
\begin{aligned}
& \alpha=\frac{\left[\Delta \log _{10}\left(F_{n}\right)\right]}{\left.\left[\Delta \log _{10}(n)\right)\right]} \\
& F_{n}=\sqrt{\frac{1}{L} \sum_{k=1}^{L}\left(y_{k}-y_{n k}\right)^{2}} \text { and } y_{k}=\sum_{i=1}^{k}\left[\left\{x_{i}\right\}-\bar{x}_{i}\right], k=1, \ldots, L .
\end{aligned}
$$

Based on our previous work (Phinyomark, Phukpattaranont, Limsakul, \& Phothisonothai, 2011d), the minimum box size $n_{\min }$ is set at four, the maximum box size $n_{\max }$ is set at one-tenth of the signal length, and the box size increment is based on a power of two. A least-square fit, which is applied to the profiles $y_{k}$, is the quadratic polynomial fit.

Higuchi method (HG) is another fractal dimension estimator (Higuchi, 1988), which performs well in the classification of surface EMG signals and the simulated signals (Arjunan \& Kumar, 2010; Esteller, Vachtsevanos, Echauz, \& Litt, 2001). For $m=1,2, \ldots, k$, a negative slope of the line relating the logarithm of the length of the curve $\left(L_{m k}\right)$ to the logarithm of the discrete time interval between points $(k)$ 
is used as a feature value $(D)$. Based on the finding of Phothisonothai and Nakagawa (2007), the maximum time interval $k_{\max }$ is set at 128 . This value is from the relationship $L_{k} \alpha k^{D}$, which can be given by

$$
\begin{aligned}
& L_{m k}=\frac{1}{k}\left\{\left(\left\lfloor\sum_{j=1}^{k}\left|x_{m+j k}-x_{m+(j-1) \cdot k}\right|\right) \cdot \frac{L-1}{\left\lfloor\frac{L-m}{k}\right\rfloor \cdot k}\right\} ;\right. \\
& X_{m}^{k}=\left\{x_{m}, x_{m+k}, x_{m+2 k}, \ldots, x_{\left.m+\mid \frac{L-m}{k}\right\rfloor \cdot k}\right\} .
\end{aligned}
$$

Integrated EMG (IEMG) is a summation of the absolute value of the EMG signal amplitude over the time segment, which can be expressed as

$$
\mathrm{IEMG}=\sum_{i=1}^{L}\left|x_{i}\right|
$$

Waveform Length (WL) is a cumulative length of the EMG waveform over the time segment. It is the IEMG of the EMG wavelength. A number of previous studies call this feature as the "wavelength", which provides simple waveform complexity information. It can be calculated by

$$
\mathrm{WL}=\sum_{i=1}^{L-1}\left|x_{i+1}-x_{i}\right|
$$

Maximum Fractal Length (MFL) is proposed as a feature, which can measure EMG signal patterns at low level muscle contraction (Arjunan, 2008). It is defined as the average fractal length of the signal measured at the smallest scale from the HG method in Eq. (6). If the smallest scale is set at one, MFL can be defined as the modification of the WL using a logarithm function as follows

$$
\text { MFL }=\log _{10}\left(\sum_{i=1}^{L-1}\left|x_{i+1}-x_{i}\right|\right) .
$$

Mean Absolute Value (MAV) is an average of the absolute value of the EMG signal amplitude. There are many ways to call this feature, e.g. averaged absolute value, average rectified value, integral of absolute value, integrated absolute value, and the first order of the $v$-Order feature. It can be given by

$$
\mathrm{MAV}=\frac{1}{L} \sum_{i=1}^{L}\left|x_{i}\right|
$$

Difference Absolute Mean Value (DAMV) is an average of the absolute value of the difference between the adjacent EMG amplitudes over the time segment (Kim, Choi, Moon, \& Mun, 2011; Park \& Lee, 1998; Yu, Jeong, Hong, \& Lee, 2012). It can be seen as the MAV of the EMG wavelength and sometimes is called as the "Average Amplitude Change (AAC)" (Fougner, 2007). It is given by 


$$
\mathrm{DAMV}=\frac{1}{L} \sum_{i=1}^{L-1}\left|x_{i+1}-x_{i}\right|
$$

Variance of EMG (VAR) is an average of the square value of the EMG signal amplitude. This is due to a mean value of surface EMG signals is close to zero, in which the equation is defined as

$$
\mathrm{VAR}=\frac{1}{L-1} \sum_{i=1}^{L} x_{i}^{2}
$$

Root Mean Square (RMS) is a square root of the average of the square of the EMG signal amplitude values. In other words, it has a similar value to the standard deviation of EMG signal amplitude. It can be defined as the modification of the VAR using a square root function as follows

$$
\mathrm{RMS}=\sqrt{\frac{1}{L} \sum_{i=1}^{L} x_{i}^{2}}
$$

Difference Absolute Standard Deviation Value (DASDV) is a square root of the average of the square of the difference between the adjacent EMG amplitudes over the time segment. It is the RMS of the EMG wavelength, as can be formulated as

$$
\operatorname{DASDV}=\sqrt{\frac{1}{L-1} \sum_{i=1}^{L-1}\left(x_{i+1}-x_{i}\right)^{2}} .
$$

Approximation Entropy (ApEn) and Sample Entropy (SampEn) are techniques used to quantify the unpredictability of fluctuations in EMG signal amplitudes over the time segment (Zhao et al., 2006a, 2006b). They provide the waveform similarity or complexity information. SampEn is developed from ApEn to avoid the bias caused by self-matching. Both methods use two input parameters: $m$ and $r$, where $m$ is the pattern length and $r$ is the criterion of similarity (Zhang \& Zhou, 2012). The basic idea is about the estimation of the conditional probability that the patterns of the EMG signal amplitude, which are similar to each other within a predefined tolerance $r$, will remain similar for the next comparison point (Richman \& Moorman, 2000).

Mean Frequency (MNF) is an average frequency, which is calculated as a sum of the product of the EMG power spectrum and the frequency divided by a total sum of the EMG power spectrum. There are other ways to call this feature, such as central frequency and the spectral center of gravity. It can be defined as

$$
\mathrm{MNF}=\sum_{j=1}^{M} f_{j} P_{j} / \sum_{j=1}^{M} P_{j}
$$

where $f_{j}$ is the frequency of the spectrum at a frequency bin $j, P_{j}$ is the EMG power spectrum at a frequency bin $j$, and $M$ is the length of whole frequency bin.

Median Frequency (MDF) is a frequency at which the EMG power spectrum is divided into two areas with an equal total power, as can be expressed as

$$
\sum_{j=1}^{\mathrm{MDF}} P_{j}=\sum_{j=\mathrm{MDF}}^{M} P_{j}=\frac{1}{2} \sum_{j=1}^{M} P_{j} .
$$




\section{Experimental Data for Extracting EMG Features}

All features are computed from the EMG data, which are recorded from 20 subjects (10 males and 10 females). The subjects were asked to perform 8 motions consisting of forearm pronation (FP), forearm supination (FS), wrist flexion (WF), wrist extension (WE), wrist radial deviation (WR), wrist ulnar deviation (WU), hand open (HO), and hand close (HC), and maintain for $2 \mathrm{~s}$. Each motion was repeated 15 trails per day for four separate days. EMG data were measured from 4 forearm muscles and an upper arm muscle: extensor carpi radialis longus (ECRL), extensor carpi ulnaris (ECU), extensor digitorum communis (EDC), flexor carpi radialis (FCR), and biceps brachii (BB). More details about data acquisition and experiments can be found in Phinyomark et al. (2011d).

To briefly explain the relationship between actions associated with surface EMG signals and EMG features, the amplitude shape of surface EMG signals acquired from all the muscles and motions from a trial in time domain is shown in Fig. 3, together with one extracted feature: MAV. It is clearly shown in the figure that the EMG amplitude shapes from all muscles are significantly different according to the direction of eight motions. For instance, we can observe that the EMG magnitudes of WE are very high in two extensor muscles, i.e., ECRL and ECU. These two muscles are the corresponding muscles to produce wrist extension, i.e., high contraction level. On the other hand, the EMG magnitudes of WF are low in both extensor muscles but are very high in the flexor muscle, FCR. As we know that the MAV feature is used to estimate the EMG amplitude/magnitude level, so MAV features of WE extracted from ECRL and ECU are higher than the other muscles. In the same way, the MAV feature of WF extracted from FCR is relatively higher than the other muscles.

Figure 3. An example of surface EMG signals in time domain from the first trail in the first day of subject 1 (5 muscles and 8 motions with rest state) and the extracted MAV features.

\section{ANTHROPOMETRIC VARIABLES Definition of Anthropometric Variables}

Anthropometry is a measurement of the dimensions of the different parts of human body. There are two types of measurement: 1) static dimension and 2) dynamic dimension. In order to use anthropometric variables to calibrate the EMG-based MCI systems, only static dimensions are considered. There are many anthropometric variables, e.g. overhead reach height and breadth, shoulder circumference and length, and waist front and back length. However, only twelve related variables are chosen for the experiments in this chapter.

\section{Computation of Anthropometric Variables}

Following is a brief description of all anthropometric variables, which were investigated and discussed for this chapter.

Body mass is measured in kilograms $(\mathrm{kg})$. It is defined as a subject's weight or mass, which can be measured by the balance type scales. A subject stands on the center of the scale platform.

Stature or standing height is measured in centimeters $(\mathrm{cm})$. It is defined as the distance from the bottom of the feet to the top of the head of a subject, which can be measured by a stadiometer. A subject stands erect on the center of the base plate with heels together, and head in the Frankfort plane.

BMI or body mass index is a roughly estimation of human body fat based a subject's weight and height in kilograms $/$ meters $^{2}\left(\mathrm{~kg} / \mathrm{m}^{2}\right)$. It can be defined as 
$\mathrm{BMI}=\frac{\text { body mass }}{(\text { stature })^{2}}$

The remaining anthropometric variables are measured in centimeters $(\mathrm{cm})$, as shown in Fig. 4, using a tape for biceps and forearm circumferences, a sliding or small bone caliper for hand breadth and length, a wide sliding torso caliper for elbow-hand grip length, elbow-fingertip length, shoulder-elbow length and bi-deltoid breadth, and a measuring block with tape measure for forward grip reach. The specific locations are defined as the following:

Figure 4. Anthropometric measurements: stature, forward grip reach, biceps circumference, forearm circumference, hand breadth, hand length, shoulder-elbow length, elbow-hand grip length, elbowfingertip length, and bi-deltoid breadth.

Biceps circumference is a linear distance around the upper arm when subject stands. The upper arm is extended forward horizontally and the elbow is flexed about $90^{\circ}$ (the arm is abducted). It is measured at the level of the drawn biceps point landmark, which is the point of maximum quiet inspiration.

Forearm circumference is a linear distance around the lower arm with the same posture as measured the biceps circumference. It is usually measured at the level of maximum forearm circumference.

For hand breadth, subject sits with right hand flats on a table and the fingers are together and straight. Hand breadth is measured from metacarpalphalangeal joint II to metacarpalphalangeal joint V.

Hand length is measured from the wrist landmark to dactylion with the same posture as the hand breadth.

Elbow-hand grip length is measured from the posterior tip of the olecranon process to the center of grip during holding a pencil. It can be called as the elbow to center of grip.

Elbow-fingertip length is measured from the posterior tip of the olecranon process to dactylion.

Shoulder-elbow length is measured from the right acromion landmark to the inferior tip of the olecranon process of the right elbow.

Forward grip reach or functional reach is measured from the back wall to the tip of the thumb.

Bi-deltoid breadth is measured across the body at the level of the deltoid landmarks.

\section{Experimental Anthropometric Data}

It should be noted that the anthropometric variables are measured in the same day for all twenty subjects from the right arm of the subject using the standard instruments (Centurion Kit, Rosscraft). Due to the significant difference of muscle size and force between male and female subjects, anthropometric variables have usually been investigated and discussed by gender: male and female (Anakwe et al., 2007; Holzbaur, Murray, Gold, \& Delp, 2007). In our experiments, the difference of anthropometric variables between male and female subjects is statistically significant at $p<0.01$ for 8 parameters (body weight, stature, biceps circumference, forearm circumference, hand breadth, hand length, elbow-fingertip length, and forward grip reach) and significant at $p<0.10$ for the remainders (BMI, elbow-hand grip length, shoulder-elbow length, and bi-deltoid breadth). In addition, to avoid the effect of ages we used the subjects from the same age, around 21 years old (no significant difference, $p=0.458$ ). Twelve anthropometric variables from 10 males and 10 females are reported respectively in Table 1 and Table 2. 
Table 1. Twelve anthropometric variables of 10 male subjects (M1-M10) with the mean and the standard deviation (SD) of each variable.

\begin{tabular}{lcccccccccccc}
\hline \multicolumn{1}{c}{ Subjects } & \multirow{2}{*}{ M1 } & \multirow{2}{*}{ M2 } & \multirow{2}{*}{ M3 } & M4 & M5 & M6 & M7 & M8 & M9 & M10 & Mean & SD \\
\hline Body weight & 49.0 & 62.0 & 65.0 & 74.0 & 73.0 & 58.0 & 57.0 & 55.0 & 63.0 & 54.0 & 61.0 & 8.1 \\
Stature & 166.5 & 170.0 & 173.0 & 177.0 & 172.0 & 170.0 & 170.0 & 167.5 & 167.0 & 164.0 & 169.7 & 3.7 \\
BMI & 17.7 & 21.5 & 21.7 & 23.6 & 24.7 & 20.1 & 19.7 & 19.6 & 22.6 & 20.1 & 21.1 & 2.1 \\
Biceps circumference & 23.1 & 26.2 & 27.5 & 32.6 & 32.4 & 30.7 & 26.1 & 23.6 & 26.7 & 25.6 & 27.5 & 3.4 \\
Forearm circumference & 22.5 & 22.4 & 25.2 & 29.1 & 28.2 & 26.8 & 23.4 & 23.1 & 24.3 & 22.7 & 24.8 & 2.5 \\
Hand breadth & 7.4 & 7.2 & 8.4 & 9.1 & 8.9 & 12.6 & 11.7 & 7.5 & 7.8 & 7.6 & 8.8 & 1.9 \\
Hand length & 17.2 & 17.8 & 15.5 & 18.2 & 18.9 & 19.1 & 19.3 & 17.4 & 18.3 & 17.1 & 17.9 & 1.1 \\
Elbow-hand grip length & 34.4 & 35.1 & 37.6 & 39.3 & 39.7 & 33.9 & 33.8 & 36.0 & 38.5 & 38.4 & 36.7 & 2.3 \\
Elbow-fingertip length & 46.8 & 49.7 & 52.2 & 52.2 & 52.6 & 46.2 & 46.2 & 47.2 & 50.0 & 49.7 & 49.3 & 2.5 \\
Shoulder-elbow length & 37.7 & 33.6 & 36.9 & 37.8 & 38.2 & 35.7 & 38.4 & 35.4 & 38.3 & 36.6 & 36.9 & 1.6 \\
Forward grip reach & 75.6 & 74.5 & 80.7 & 89.0 & 80.4 & 74.3 & 75.7 & 72.6 & 83.5 & 78.1 & 78.4 & 5.0 \\
Bi-deltoid breadth & 39.3 & 46.4 & 44.6 & 54.7 & 47.3 & 37.4 & 30.4 & 42.1 & 45.9 & 47.4 & 43.6 & 6.7 \\
\hline
\end{tabular}

Table 2. Twelve anthropometric variables of 10 female subjects (F1-F10) with the mean and the SD of each variable.

\begin{tabular}{lcccccccccccc}
\hline \multicolumn{1}{c}{ Subjects } & \multirow{2}{*}{ F1 } & \multirow{2}{*}{ F2 } & \multirow{2}{*}{ F3 } & \multirow{2}{*}{ F4 } & \multirow{2}{*}{ F5 } & \multirow{2}{*}{ F6 } & F7 & \multirow{2}{*}{ F8 } & F9 & \multirow{2}{*}{ F10 } & Mean & SD \\
\hline Variables & 47.0 & 45.0 & 53.0 & 46.0 & 54.0 & 45.0 & 43.0 & 56.0 & 50.0 & 49.0 & 48.8 & 4.4 \\
Body weight & 150.0 & 160.0 & 156.0 & 155.0 & 160.0 & 146.0 & 159.0 & 163.0 & 167.0 & 162.0 & 157.8 & 6.3 \\
BMI & 20.9 & 17.6 & 21.8 & 19.1 & 21.1 & 21.1 & 17.0 & 21.1 & 17.9 & 18.7 & 19.6 & 1.8 \\
Biceps circumference & 24.4 & 19.8 & 27.6 & 24.2 & 24.8 & 25.5 & 22.6 & 24.9 & 22.3 & 21.5 & 23.8 & 2.2 \\
Forearm circumference & 21.6 & 19.1 & 22.9 & 21.5 & 22.2 & 21.9 & 19.7 & 22.7 & 20.6 & 20.9 & 21.3 & 1.2 \\
Hand breadth & 7.4 & 6.4 & 7.3 & 7.5 & 6.9 & 6.6 & 6.9 & 8.1 & 7.4 & 7.2 & 7.2 & 0.5 \\
Hand length & 15.6 & 16.7 & 16.8 & 16.6 & 16.4 & 14.7 & 16.1 & 15.2 & 17.1 & 17.2 & 16.2 & 0.8 \\
Elbow-hand grip length & 32.8 & 35.0 & 34.8 & 34.6 & 34.4 & 32.6 & 34.3 & 35.2 & 38.4 & 36.2 & 34.8 & 1.6 \\
Elbow-fingertip length & 43.3 & 46.8 & 46.4 & 46.4 & 46.2 & 39.1 & 46.2 & 47.1 & 49.0 & 47.7 & 45.8 & 2.8 \\
Shoulder-elbow length & 34.9 & 35.3 & 36.1 & 35.8 & 33.1 & 34.2 & 33.0 & 37.1 & 37.8 & 36.0 & 35.3 & 1.6 \\
Forward grip reach & 65.1 & 72.0 & 60.8 & 68.1 & 75.7 & 65.2 & 70.2 & 63.3 & 74.4 & 70.6 & 68.5 & 4.9 \\
Bi-deltoid breadth & 41.9 & 36.8 & 43.4 & 41.2 & 41.0 & 37.8 & 36.9 & 37.0 & 37.2 & 38.7 & 39.2 & 2.5 \\
\hline
\end{tabular}

\section{STATISTICAL ANALYSIS}

Correlation analysis is used as an evaluating function in the study. It measures a relationship between two parameters and provides a statistic known as the correlation $r$ coefficient. This coefficient shows the degree of a linear relationship between two measured variables. Correlation coefficients can interpret as the weak or low correlation when $r \leq 0.35$, the modest or moderate correlation when $0.35<r \leq 0.67$, and the strong or high correlation when $0.67<r \leq 1$ (Taylor, 1990). Actually, the $r$ value contains both a magnitude and a direction (positive and negative) of the relationship. However, in our experiment only the magnitude of correlation coefficient (the absolute value of average $r$ coefficient) is used. Moreover, due to a small number of subjects $n$ (less than 20), the significant level is set at $p<0.05$ and is tested using $t$-test, as can be defined by

$$
t=r \sqrt{\frac{n-2}{1-r^{2}}} .
$$


It should be noted that degrees of freedom for entering the $t$-distribution is defined as $n-2$. Only the combinations between EMG features extracted from a muscle and anthropometric variables that have the strong and significant correlations in at least 4 motions are considered for the next stage.

\section{RELATIONSHIP BETWEEN EMG FEATURE AND ANTHROPOMETRIC VARIABLE}

To reach the high potential of EMG-based MCIs in the context of real-world requirements, the proposed anthropometric variables have been investigated the relationship with robust EMG features, which are divided into 4 groups as follows:

(1) Noise tolerance: WAMP, MYOP, SSC, and ZC;

(2) Low-level and high-level surface EMG signals: DFA, HG, MFL, DAMV, DASDV, WL, VAR, IEMG, MAV, and RMS;

(3) Fluctuating EMG signals over time: SampEn and ApEn;

(4) Muscle fatigue: MNF and MDF.

It should be noted that the relationship between anthropometric variables and the Hudgins' time domain feature set, i.e. SSC, ZC, WL, MAV and mean absolute value slope, is presented and discussed in detail in our previous study (Phinyomark et al., 2013b).

\section{Feature Set 1: Noise Tolerance}

Noise is one of the major problems in the analysis of surface EMG signals. Zardoshti-Kermani et al. (1995) proposed that the robustness of EMG features is one of the three major properties for evaluating high-quality features. In their study, the definition of robustness is limited to the tolerance of noise. Artificial white noise at different noise levels was used to evaluate the robustness of features. The cluster separability of feature spaces should be preserved as much as possible in a noisy environment. This property is important because both biological and environmental resources can generate noise and artifact. It is difficult to record only a pure signal component without noise. For this reason, noise removal or noise reduction is an important step before performing feature extraction in most of MCI systems. Many noise removal tools have been proposed, e.g. notch filter, band pass filter, adaptive filter, and wavelet filter (Jindapetch, Chewae, \& Phukpattaranont, 2012; Phinyomark, Phukpattaranont, \& Limsakul, 2011c). Unfortunately, there are not any filters that can remove one hundred percent of noise. Sometimes it removes some important parts of real EMG signals (De Luca, Gilmore, Kuznetsov, \& Roy, 2010; Li, Li, $\mathrm{Yu}, \&$ Geng, 2011). So EMG features that have high tolerance for biological and environmental noises should increase the ability of EMG-based gesture recognition.

Noises contaminated in surface EMG signal can be categorized into four major types: 1) ambient noise, 2) motion artifact, 3) the inherent instability of surface EMG signals, and 4) the inherence in electronic components in the detection and recording equipment (De Luca, 2002; Reaz, Hussain, \& Mohd-Yasin, 2006). The first three types have a specific band of frequencies, but the last noise type has a broaden frequency band and falls in a usable energy band of surface EMG signals. In the literature, four noisy EMG signals that consist of baseline noise, movement artifact, power-line interference, and random noise, i.e., white Gaussian noise, have been simulated and used to develop robust EMG methods (Phinyomark, Phukpattaranont, \& Limsakul, 2012f). First two simulated noises, i.e., baseline noise and movement artifact have a narrow frequency band below $20 \mathrm{~Hz}$, which ranges outside the EMG energy band. We can use a high-pass filter at a cut-off frequency of $20 \mathrm{~Hz}$ to remove these kinds of noise (De Luca et al., 2010). On the other hand, power-line interference $(50 / 60 \mathrm{~Hz})$ and random noise $(0-1000 \mathrm{~Hz})$ have a frequency band in a range of usable EMG energy band $(20-500 \mathrm{~Hz})$, therefore such noises can be only reduced to a predictable level or cannot be entirely removed. 
In our previous works (Phinyomark, Limsakul, \& Phukpattaranont, 2008, 2009), nine time domain and frequency domain features: RMS, WL, ZC, SSC, WAMP, HIST, AR, MDF, and MNF, were evaluated the robust performance based on two noise types: $50-\mathrm{Hz}$ interference and white Gaussian noise, at different signal-to-noise ratios (0-20 dB SNRs). The experimental results showed that, on average WAMP is the most robust feature, followed by ZC and SSC features. These features are in the same feature group, together with MYOP, which compute in time domain and provide frequency information (Phinyomark, Phukpattaranont, \& Limsakul, 2012c). All features in this group have a threshold parameter, which can be used to avoid some noises (Hudgins et al., 1993). In addition, the distribution of feature spaces in this group is similar to each other, particularly for WAMP and ZC.

Table 3. Correlation coefficients $|r|$ between anthropometric variables and EMG features (WAMP, MYOP, SSC, and ZC) in cases of strong and significant relationships (at least 4 movements for a muscle) based on 10 males and/or 10 females.

\begin{tabular}{lllllr}
\hline Feature & Gender & $\begin{array}{l}\text { Anthropometric } \\
\text { variable }\end{array}$ & $\begin{array}{l}\text { Muscle } \\
\text { position }\end{array}$ & Movements & Average $|r|($ min-max) \\
\hline WAMP & Male & Bi-deltoid breadth & ECU & FP, WE, WF, WU, HC & $0.75(0.72-0.78)$ \\
& Female & Bi-deltoid breadth & BB & FP, FS, WF, WR & $0.72(0.69-0.77)$ \\
MYOP & Male & Bi-deltoid breadth & ECU & FP, WE, WF, WU, HC & $0.77(0.76-0.77)$ \\
& Female & Bi-deltoid breadth & BB & FP, WF, WR, WU & $0.72(0.68-0.81)$ \\
SSC & Female & Bi-deltoid breadth & BB & FP, FS, WF, WU, HC & $0.80(0.70-0.86)$ \\
ZC & Male & Biceps circumference & BB & FP, FS, WF, HO & $0.76(0.69-0.85)$ \\
& Female & Bi-deltoid breadth & BB & FP, FS, WF, WU, HC & $0.77(0.70-0.84)$ \\
\hline
\end{tabular}

From Table 3, all four features extracted from the BB muscle have a strong relationship with the bideltoid breadth for female subjects. For male subjects, there are two relationships: 1) WAMP and MYOP from the ECU muscle and bi-deltoid breadth, and 2) ZC from the BB muscle and biceps circumference. All relationships share the motions of FP and WF.

Although the SSC feature has a higher average $|r|$ value than other three features for female subjects, it does not have a strong relationship with any anthropometric variables for male subjects. SSC and ZC are the members of the Hudgins' time domain feature set (Hudgins et al., 1993) and their classification performances are not influenced by the variation in muscle contraction effort (Tkach et al., 2010). Other features in Hudgins' time domain feature set are MAV, WL, and mean absolute value slope. Chan et al. (2000) re-evaluated the classification performance of the Hudgins' feature set using fuzzy approach. The SSC feature does not improve classification performance and in some subjects the classification accuracy decreases. So SSC is not recommended for developing robust MCI system.

Based on the similar distribution of feature spaces of ZC and WAMP (Phinyomark et al., 2012c), one of them should be selected in order to avoid the information redundancy in an EMG feature vector. The selection depends on the choice of the system. If the automatically calibrated system is preferred, the ZC feature is recommended. On the other hand, if the semi-automatically calibrated system is acceptable, WAMP is a suitable feature. WAMP and MYOP have the same associations with the anthropometric variables, whereas WAMP is the most robust feature against noises. Moreover, WAMP has higher classification accuracy than other three features in the classification of upper-limb motions based on a robust linear discriminant analysis (LDA) classifier with and without periodic retraining scheme (Phinyomark et al., 2011a, in press b). WAMP is also successful in the classification of lower-limb exercise activities for the elderly (Phinyomark et al., 2012a) and in the estimation of muscle force levels (Kamavuako et al., 2013).

\section{Feature Set 2: Low-level and High-level Surface EMG Signals}


If the low-noise EMG signals are obtained from an EMG recording system with/without noise removal algorithms, the next challenge in EMG-based gesture recognition is about weak measured EMG signals. Simple MCI systems use the potential of a large difference between a high EMG magnitude at strong muscle contractions and a very low magnitude (close to zero) at the rest of the contractions. EMG amplitude estimators, i.e., RMS and MAV are sufficient enough for classifying the proposed activities (Clancy \& Hogan, 1999). However, such systems often offer a few control schemes with a single speed of actuation based on a few EMG channels. To increase the number of control commands, a variety of motions and EMG channels have been proposed for the recognition system. In that case, it is not possible to get only strong EMG signals. Weak or low-level EMG signals (low SNR signals) must be measured. There are two cases: 1) a weak EMG signal is measured from a major corresponding muscle when a user performs a low force motion or a little movement (Arjunan, 2008) and 2) a weak EMG signal is measured from a minor corresponding muscle when a user performs a high force motion or a strong movement (Phinyomark, Phukpattaranont, \& Limsakul, 2012d).

To deal with the analysis of low-level EMG signals, a fractal analysis is used. Fractals refer to signal patterns, which exhibit self-similarity, that have fractal dimension. Fractal dimension of surface EMG signals is found under different types and levels of muscle contraction using several fractal methods, e.g. box-counting method (Gupta, Suryanarayanan, \& Reddy, 1997), correlation dimension method (Hu, Wang, \& Ren, 2005), critical exponent analysis (Phinyomark, Phothisonothai, Phukpattaranont, \& Limsakul, 2011b), and Katz method (Gitter \& Czerniecki, 1995). Based on the finding in previous studies, a fractal dimension of EMG signals depends on the level of muscle contraction during strong or high level activities (Gupta et al., 1997; Hu et al., 2005). For the low level of muscle contraction, fractal dimension does not change with change in the level of muscle contraction. It is a measure of the size and complexity of the muscles (Arjunan, 2008).

In our previous work (Phinyomark et al., 2012d), a fractal dimension estimator DFA was examined the performance in the classification of low-level EMG signal patterns compared with another fractal estimator HG (Arjunan \& Kumar, 2010). The experimental results showed that, DFA has better cluster separability than HG (Phinyomark et al., 2011d), as well as classification performance (Phinyomark et al., 2012d). On the other hand, features based on a magnitude detector provide better performance in the classification of high-level EMG signal patterns than fractal features. So a combination of fractal and magnitude features is recommended (Arjunan \& Kumar, 2011).

The past several years, magnitude features extracted from the first-order difference of EMG time series, i.e., MFL, DAMV, DASDV, and WL, present higher accuracy than magnitude features extracted from the original time series, i.e., VAR, IEMG, MAV, and RMS (Brzostowski \& Zieba, 2011; Kim et al., 2011; Kim, Jeong, Lee, \& Song, 2012; Yu et al., 2012). In addition, the distribution of feature spaces in both groups is similar to each other for some motions and muscles.

Table 4. Correlation coefficients $|r|$ between anthropometric variables and EMG features (DFA, HG, MFL, DAMV, DASDV, WL, VAR, IEMG, MAV, and RMS) in cases of strong and significant relationships (at least 4 movements for a muscle) based on 10 males and/or 10 females.

\begin{tabular}{llllll}
\hline \multirow{2}{*}{ Feature } & Gender & $\begin{array}{l}\text { Anthropometric } \\
\text { variable }\end{array}$ & $\begin{array}{l}\text { Muscle } \\
\text { position }\end{array}$ & Movements & Average $|r|(\min -\max )$ \\
\hline DFA & Female & Stature & FCR & FP, WF, WR, WU, HO & $0.74(0.67-0.83)$ \\
& Female & Elbow-fingertip length & FCR & WF, WU, HO, HC & $0.73(0.68-0.79)$ \\
HG & Male & BMI & EDC & FP, WU, HO, HC & $0.72(0.67-0.81)$ \\
\hline MFL & Male & Bi-deltoid breadth & ECU & FP, WE, WF, WU, HC & $0.75(0.70-0.78)$ \\
& Both & Bi-deltoid breadth & BB & WE, WR, WU, HC & $0.69(0.68-0.69)$ \\
& Female & Bi-deltoid breadth & BB & FP, FS, WF, WR, WU, HC & $0.76(0.70-0.78)$ \\
DAMV & Male & Bi-deltoid breadth & ECU & FP, WE, WF, WU, HC & $0.72(0.67-0.75)$
\end{tabular}




\begin{tabular}{llllll} 
& Male & Bi-deltoid breadth & BB & WE, WR, WU, HC & $0.69(0.68-0.70)$ \\
& Female & Bi-deltoid breadth & BB & FP, FS, WR, HC & $0.73(0.68-0.78)$ \\
DASDV & Male & Bi-deltoid breadth & ECU & FP, WE, WF, WU & $0.72(0.67-0.75)$ \\
& Male & Bi-deltoid breadth & BB & WE, WR, WU, HC & $0.70(0.70-0.71)$ \\
& Female & Bi-deltoid breadth & BB & FP, FS, WF, WR & $0.72(0.67-0.75)$ \\
WL & Male & Bi-deltoid breadth & BB & WE, WR, WU, HC & $0.69(0.68-0.70)$ \\
& Female & Bi-deltoid breadth & BB & FP, FS, WF, WR, HC & $0.75(0.72-0.83)$ \\
\hline VAR & Male & Bi-deltoid breadth & ECU & FP, WE, WF, HC & $0.71(0.68-0.76)$ \\
IEMG & Male & Bi-deltoid breadth & ECU & FP, WE, WF, WU, HC & $0.77(0.73-0.82)$ \\
MAV & Male & Bi-deltoid breadth & ECU & FP, WE, WF, WU, HC & $0.77(0.73-0.82)$ \\
RMS & Male & Bi-deltoid breadth & ECU & FP, WE, WF, WU, HC & $0.76(0.71-0.82)$ \\
\hline
\end{tabular}

Features in Table 4 can be divided into three feature groups based on the explanation above. Due to a large difference of feature spaces of the fractal features compared to the magnitude features, anthropometric variables that have strong associations with fractal features are different to other magnitude features. DFA computed from the FCR muscle has a strong relationship with the stature and elbow-fingertip length for female subjects. On the other hand, HG computed from the EDC muscle has a strong relationship with the BMI for male subjects. Both DFA and HG features estimate the fractal dimension of surface EMG signals, so the selection of fractal features is dependent on the gender of target users. On average DFA is more accurate than HG about 2\%-5\% (Phinyomark et al., 2012d).

For magnitude features, strong associations are found only with the bi-deltoid breadth. For female subjects, only features extracted from the BB muscle have a strong relationship with the variable. All relationships share the motions of FP, FS, and WR. For male subjects, two muscles have the strong relationships: ECU and BB muscles. Although feature space distribution of features extracted from the original and the first-order difference of EMG signals is similar to each other for some motions and muscles, the best feature from each group is usually selected in many previous studies to be a member of an optimal multiple feature set based on the feature selection scheme (Phinyomark et al., 2013b).

For the first-order differencing magnitude features, MFL outperforms others, followed respectively by DAMV, DASDV, and WL, based on many state-of-the-art classifiers, i.e., the LDA, the quadratic discriminant analysis (QDA), the artificial neural network (ANN), the k-nearest neighbor (k-NN), and the maximum likelihood estimation (MLE), with and without the periodic retraining scheme (Arjunan \& Kumar, 2010; Kim et al., 2011; Phinyomark et al., 2013b; Yu et al., 2012). Although WL performs the best in accuracy, stability, and computation load among the single features in several previous studies (Oskoei \& Hu, 2008; Phinyomark et al., 2010), it is because of its popularity and other first-order differencing features were not included in the evaluating studies. In total, MFL is recommended to be used as a representative feature in this group instead of WL.

For the original magnitude features, all features have the same relationship with the anthropometric variable. Due to its similarity of IEMG to MAV and its similarity of VAR to RMS, the IEMG and VAR features should be excluded, because in comparison IEMG and VAR result in weaker performance in classification than MAV and RMS (Oskoei \& Hu, 2008; Phinyomark et al., 2012c). For MAV and RMS, both features are close together in the success rate of classification, as well as the computational cost. The selection of MAV and RMS should be based on the probability density function (PDF) of the measured EMG signals. If the EMG PDF is close to the Gaussian density, an optimal EMG amplitude estimator is RMS (Hogan \& Mann, 1980) based on both theoretically and experimentally. On the other hand, if the EMG PDF is close to the Laplacian density, an optimal EMG amplitude estimator is MAV (Clancy \& Hogan, 1999). Moreover, MAV and RMS performance in the estimation of muscle force is similar to the performance of WAMP and WL features (Kamavuako et al., 2013). 


\section{Feature Set 3: Fluctuating EMG Signals over Time}

Multifunction EMG pattern recognition systems that allow high recognition rate in research laboratories are usually based in single-session experiments. Most of related works collected EMG data only from a single day. A next major problem is about the fluctuation of surface EMG signals over time. It is widely known that EMG signals measured in one day are relatively different from the EMG signals measured in another day, even on the same muscle and also same subject (Jain et al., 2012; Kaufmann, Englehart, \& Platzner, 2010). This can be due to many factors, such as the EMG electrode location shift, the variation in muscle contraction effort, or change in the architecture characteristics of the subject (Saponas et al., 2010; Tkach et al., 2010). So it is possible that features extracted from the initial training data (one day) and the present testing data (another day) are significant difference, which will degrade the performance of the pattern matching algorithms. In order to implement the MCIs based on EMG pattern recognition in clinics, the robustness over time of the system (i.e., feature extraction and classification algorithm) is very important.

In the literature, however, this problem has rarely been evaluated. Sensinger et al. (2009) and Jain et al. (2012) found that the performance of the EMG pattern recognition systems can degrade within hours after initial classifier training. Zhang et al. (2007) studied the effect of the number of days used as the training data sets. They found that if the data used for training the classifier decrease, the classification accuracy also decreases based on EMG signals recorded from five different days. Kaufmann et al. (2010) evaluated the performance of five state-of-the-art classifiers: support vector machine (SVM), decision tree (DT), the ANN, k-NN, and LDA, in the classification of surface EMG signals recorded from 21 days. They also found that the performance of the EMG pattern recognition systems degrades with increasing time difference between initial classifier training and testing data for all studied classifiers (about 8\%-15\%), except the LDA (about 3.6\%) using the Hudgins' time domain feature set.

Using the same EMG data recorded during 21 days, fifty state-of-the-art EMG features in time domain and frequency domain were evaluated in one of our previous works (Phinyomark et al., 2013b). Among the single features, SampEn performed the highest classification accuracy (93.37\%) by training the LDA classifier using EMG from only the first day without any retraining classifier again, followed by ApEn (84.68\%) and MFL (82.07\%). The EMG data were recorded from four surface EMG channels on the forearm for 21 days with 5-6 trials per day, and in each trial 11 wrist and finger motions were performed (Kaufmann et al., 2010).

SampEn and ApEn features extracted from the FCR muscle have a strong relationship with the forward grip reach for female subjects. Both relationships share the four motions of FP, FS, HO, and HC. In addition to the robustness over time, SampEn can use to detect the onset of EMG activity while suppressing spurious background spikes (Zhang \& Zhou, 2012).

Table 5. Correlation coefficients $|r|$ between anthropometric variables and EMG features (SampEn, ApEn) in cases of strong and significant relationships (at least 4 movements for a muscle) based on 10 males and/or 10 females.

\begin{tabular}{llllll}
\hline Feature & Gender & $\begin{array}{l}\text { Anthropometric } \\
\text { variable }\end{array}$ & $\begin{array}{l}\text { Muscle } \\
\text { position }\end{array}$ & Movements & Average $|r|(\min -\max )$ \\
\hline SampEn & Female & Forward grip reach & FCR & FP, FS, WU, HO, HC & $0.75(0.69-0.86)$ \\
ApEn & Female & Forward grip reach & FCR & FP, FS, HO, HC & $0.76(0.67-0.89)$ \\
\hline
\end{tabular}

\section{Feature Set 4: Muscle Fatigue}

Muscle fatigue is generally resulted when a user performs the repetitive proposed motions for a long time and cannot produce the certain level of force with the muscle (De Luca, 1984). Generally, muscle fatigue results in an increase in EMG signal amplitude and a downward shift of EMG frequency spectrum 
(Cifrek, Medved, Tonković, \& Ostojić, 2009). However, Tkach et al. (2010) reported that the effect of muscle fatigue on the classification performance of eleven time domain features (i.e., WAMP, ZC, SSC, WL, VAR, MAV, v-Order, log-Detector, EMG histogram, autoregressive coefficients, and cepstrum coefficients) is very weak. On the other hand, for frequency domain features, MNF and MDF have been hailed so far as the gold standard for the muscle fatigue detection with surface EMG signals (Al-Mulla, Sepulveda, \& Colley, 2011).

The experimental results showed that MDF does not have a strong relationship with any anthropometric variables, whereas MNF extracted from the FCR muscle of female subjects has a strong relationship with the hand length for four motions: FS, WU, HO, and $\mathrm{HC}$ at the average correlation coefficient $|r|$ of 0.76 (in range of 0.71-0.87). Further, MNF performs better performance in the classification of upper-limb motions than MDF and other frequency domain features, i.e., peak frequency, frequency ratio, power spectrum ratio, and the variance of central frequency (Phinyomark et al., 2012c, 2013b). The modified MNF can also be used to estimate muscle force like time domain features (Phinyomark et al., 2012g; Thongpanja, Phinyomark, Phukpattaranont, \& Limsakul, 2013). If EMG frequency information is needed in developing EMG-based MCIs, the MNF feature is recommended to be used as an optimal frequency domain feature for both the classification of actions-based EMG signal and the assessment of muscle fatigue during actions.

\section{SEMI-AUTOMATIC AND AUTOMATIC CALIBRATION SYSTEMS}

As mentioned in the Introduction section, we can use the anthropometric variables to calibrate the systems in two different ways: a weighting factor for a classifier and a normalizing value for EMG features. Both ways can be used for adapting the system automatically. However, the difference between semi-automatic and automatic calibration systems is about the way to measure anthropometric variables. From twelve proposed anthropometric variables, only two variables: forearm and biceps circumferences can measure directly from an EMG measuring armband device, which is composed of surface EMG electrodes and the circumference measurement (Cannan \& Hu, 2011). They can be used as calibrated inputs for an automatic calibration system. On the other hand, the remaining variables cannot measure directly from the EMG measuring armband device. These variables need to be measured manually, so they can be used as calibrated inputs for a semi-automatic calibration system. From the experiments, 7 out of the 12 proposed variables have strong relationships with EMG features, which can be divided into two groups: 1) the bideltoid breadth, stature, elbow-fingertip length, BMI, forward grip reach, and hand length for a semiautomatic calibration system, and 2) the biceps circumference for an automatic calibration system.

Among the seven variables, the bi-deltoid breadth has more associations with EMG features than other variables. It can be used to calibrate many robust EMG features consisting of WAMP, MFL, MAV, and RMS. The use of a feature vector of WAMP, MFL, and MAV/RMS would result in the high classification accuracy under robust conditions such as noisy environment, variation in muscle contraction effort, and low-and-high-level muscle contractions. For instance, using this EMG multiple feature set produced $89.97 \pm 7.34 \%$ accuracy when classified the EMG data recorded from 21 days without the retraining classifier scheme (Kaufmann et al., 2010) and only the bi-deltoid breadth variable is used to calibrate the recognition system. However, if the system is not limited by a low processor, the multiple robust feature sets with respect to all studied robust conditions, which consisted of some robust features: WAMP, MFL, MAV/RMS, DFA, SampEn, and MNF, would result in high classification accuracy and could be used across users by calibrating with the correspond anthropometric variables. Hence, in future works, the stability of the possible multiple feature sets with respect to several studied robust conditions should be evaluated their performance together with the development of calibration techniques using the correspond anthropometric variables. 
On the other hand, only a combination of the ZC and the biceps circumference has a strong relationship, which can be used for an automatic calibration system. However, due to a rapid increased number of EMG wearable devices and a success of classifying motions associated with surface EMG recorded from the armband, new EMG features and other useful motions/muscles should be evaluated their associations with forearm and biceps circumferences in future studies. In addition to both the circumferences, hand circumference may be a useful anthropometric variable, because it is possible to record surface EMG signals using electrode sensor ring around the hand. This variable was also found that it has a strong relationship with the maximal grip strength and can use to create a simple model to predict the maximal grip strength ( $\mathrm{Li}$ et al., 2010). In order to explore the potential use of automatic calibration systems, a review of the EMG pattern recognition using electrode arm bands is presented in the following subsection.

\section{EMG sensor arm bands}

To measure wrist and finger actions associated with surface EMG signals, traditionally, multiple surface electrodes need to be placed right above all the corresponding muscles. However, it is difficult to identify the exact position of the muscles (Kendall et al., 2012; Vigreux, Cnockaert, \& Pertuzon, 1979). Because users typically have no detailed knowledge of human anatomy hence the EMG system is limited to only medical applications, and several muscles are closely together so it is not possible to acquire EMG from some specific single muscles. Moreover, the placing electrodes in this way require more time consuming. Errors in electrode placement degrade the classification accuracy of EMG-based MCIs (Tkach et al., 2010; Young et al., 2011, 2012).

Recent studies have designed and developed multichannel sensor rings/armbands to solve this problem. In this way, end-users will not have expertise and time to work on the electrode placement. It can be clearly seen from a recent commercial EMG sensor armband (i.e., MYO armband, see www.getmyo.com). The MYO armband is composed of a large amount of small active electrodes in a form of sensor ring and can transfer the EMG data over a wireless network, which is feasible for use outside the laboratory. This device is easy to set up and configure by a user, and provides an always-available and highly personalized input interface with is a low cost (inexpensive). It could be like a watch, wristband, jewelry, or concealed beneath clothing in the near future, which is unobtrusive. Many wireless EMG systems have also become commercially available, such as ZeroWire EMG (see www.aurion.it), BTS FREEEMG 100RT/300 (see www.btsbioengineering.com), Wave Plus EMG (see www.cometasystems.com), and MYON 320 (see www.myon-prophysics.ch).

There are two useful positions on the forearm that have been proposed in related works on EMG pattern recognition:

(1) Lower forearm positions, i.e., around the wrist (Rhee, You, \& Shin, 2011; Tang, Liu, Lv, \& Sun, 2012; You, Rhee, \& Shin, 2010, 2011);

(2) Upper forearm positions, i.e., below the elbow (Andews, Morin, \& McLean, 2009; Benko et al., 2009; Du, Lin, Shyu, \& Chen, 2010; Du, Shyu, \& Hu, 2006; Khushaba \& Kodagoda, 2012; Khushaba et al., 2013; Mogk \& Keir, 2003; Saponas et al., 2008; Shyu, Chen, Tatn, \& Hu, 2002; Smith, Huberdeau, Tenore, \& Thakor, 2009; Smith et al., 2008; Tenore et al, 2007, 2009).

For the placement of the EMG electrodes around the forearm, there are two possible ways:

(1) An adapted scheme, i.e., the distances between every two channels of electrodes are changed and dependent on the forearm sizes of the users (Andews et al., 2009; Benko et al., 2009; Saponas et al., 2008; Tang et al., 2012); 
(2) A fixed scheme, i.e., the distances between every two channels of electrodes are fixed, except one pair of electrodes in which the distance is varied depending on forearm circumference, e.g. one pair across the ulnar border (Du et al., 2006, 2010; Mogk \& Keir, 2003; Shyu et al., 2002; Smith et al., 2008, 2009). In this scheme, however, there is no guarantee that electrodes are placed over the same muscles in all users.

For lower forearm positions, the EMG electrodes are designed to place on one side of the forearm: anterior forearm or posterior forearm, as a half wristband. In You et al. $(2010,2011)$ and Rhee et al. (2011), four EMG channels are placed on the anterior side of the forearm (flexor muscles). The recognition system is used to classify all five single finger motions and three multi-finger motions (indexmiddle fingers, middle-ring fingers, and hand close) and provides the average accuracy of about $95 \%$ 97\%. On the other hand, Tang et al. (2012) used six EMG channels placed on the posterior side of the forearm (extensor muscles) instead of the anterior forearm to recognize three single finger motions (thumb, index finger, middle finger), and eight multi-finger motions. The success recognition rate is higher than $89 \%$. Tang et al. (2012) mentioned that six channels are enough to cover the circumference of the posterior side and cover all useful extensor muscles: extensor digitorum, extensor pollicis longus and brevis, extensor indicis, and extensor digiti minimi.

For upper forearm positions, the EMG electrodes are designed to place on both sides of the forearm. The number of EMG channels placed in a narrow uniform band/ring, which is proposed in the literature, is seven sensors (Du et al., 2006, 2010; Mogk \& Keir, 2003; Shyu et al., 2002) and eight sensors (Andrews et al., 2009; Khushaba \& Kodagoda, 2012; Khushaba et al., 2013; Saponas et al., 2008; Smith et al., 2009). When multiple sets of sensors were placed around the forearm, reasonable EMG signals with useful information could be acquired from the armband even the sensors are only approximately placed (Saponas et al., 2008). The landmark sensor armband is placed below the elbow in several conditions, e.g. approximately one third of the distance from the proximal end of a line from medial epicondyle to the distal head of the radius (Andrews et al., 2009; Mogk \& Keir, 2003), or approximately two inches or five centimeters below the elbow (Du et al., 2006, 2010; Shyu et al., 2002; Smith et al., 2009). For the landmark of the starting electrode pair, there are several ways, such as placing just superior to the ulna (Andrews et al., 2009) or over the flexor carpi radialis (Mogk \& Keir, 2003). On average, the proposed systems achieve more than $90 \%-95 \%$ accuracy.

Tenore et al. $(2009,2007)$ reported that there is no statistically significant difference $(p<0.05)$ in the classification accuracy using 32 electrodes placed on upper and lower forearm (6 sensor rings) and 19 electrodes placed on only upper forearm ( 3 sensor rings). The accuracy in classifying ten single flexed and extended finger motions is greater than $90 \%$ even in a transradial amputee. It means that the multichannel sensor ring may be a kind of redundant sensor. Mogk and Keir (2003) found that the EMG signal amplitudes between adjacent electrode pairs ( $3 \mathrm{~cm}$ apart) share $40 \%$ common signal (or crosstalk), and the common signal is reduced to about $10 \%$ at $6 \mathrm{~cm}$ spacing and $2.5 \%$ at $9 \mathrm{~cm}$, while only $2 \%$ common signal or crosstalk is found between flexor and extensor electrode pairs. On the other hand, we could place a similar sensor band on the other forearm to provide even more input possibilities (Saponas et al., 2008).

\section{CONCLUSIONS AND FUTURE WORKS}

This chapter presents the relationships between robust EMG features and twelve related anthropometric variables for MCIs. In feature extraction view point, the robust EMG feature is recommended for each feature set $1-4$, which is based on robust conditions in the context of real-world requirements. It can be summarized, as the following: 
(1) WAMP is the most robust feature against a variety of noises, i.e., random noise and power-line interference, followed by ZC. WAMP is also an optimal feature to estimate muscle force. It has a strong relationship with bi-deltoid breadth.

(2) DFA, a fractal (complexity) feature, is the suitable feature for the classification of low-level EMG signals, and MFL and MAV/RMS are the optimal magnitude features for the classification of high-level EMG signals. DFA has a strong relationship with the stature and the elbow-fingertip length variables, whereas MFL and MAV/RMS have strong relationships with bi-deltoid breadth.

(3) SampEn is the most robust feature for the variability of the muscle contraction over time. It is not only reliable for long-term usage but also for noise tolerance, i.e., spurious background spikes. SampEn has a strong relationship with forward grip reach.

(4) MNF is used as a standard muscle fatigue detector. It is reliable for a noisy environment and can provide additional information in frequency domain. It has a strong relationship with hand length.

The optimal robust features mentioned above have strong relationships with several experimental anthropometric variables, i.e., biceps circumference, bi-deltoid breadth, standing height, elbow-fingertip length, forward grip reach, and hand length. These variables can be used to calibrate the MCI systems in two ways. The first way is a manual calibrated input system or a semi-automatic calibration, which can be implemented using all anthropometric variables. In this way, the system can develop using many robust features, for instance, a robust multiple EMG feature set may consist of WAMP, DFA, MFL, MAV/RMS, SampEn, and MNF, and each feature can be calibrated by its related anthropometric variables. The second way is as an auto-calibration system. Based on this condition, forearm and bicep circumferences are two out of twelve variables that can be measured directly from wearable EMG device and used automatically to adapt the recognition system. However, in our experiments (eight upper-limb motions and five muscle positions) only the ZC feature has a strong and significant relationship with the biceps circumference. In future works, new features and/or other motions and muscles should be evaluated their associations with anthropometric variables.

In addition to the measured anthropometric data, we mentioned in the Introduction Section that that it possible to get the estimated anthropometric data from the published anthropometric tables. So in future works the estimated anthropometric data should be evaluated their relationships with EMG features too. If there are the strong and significant relationships between them, it means that we can add the anthropometric tables into the classification system and use there information to calibrate the system without measuring the anthropometric variables directly from the user. Further, the calibration techniques using the anthropometric variables: a weighting factor for a classifier and/or a normalizing value for EMG features should be developed and evaluated in future works. A simple idea for using anthropometric variables as a normalizing value for EMG features can be found in our preliminary study (Phinyomark et al., 2013a).

\section{ACKNOWLEDGMENT}

This work is funded by the FI MSTIC University Joseph Fourier Grenoble 1 (TIGRE project).

\section{REFERENCES}

Al-Mulla, M. R., Sepulveda, F., \& Colley, M. (2011). A review of non-invasive techniques to detect and predict localised muscle fatigue. Sensors, 11(4), 3545-3594.

Anakwe, R. E., Huntley, J. S., \& Mceachan, J. E. (2007). Grip strength and forearm circumference in a healthy population. Journal of Hand Surgery (European Volume), 32(2), 203-209.

Andews, A., Morin, E., \& McLean, L. (2009). Optimal electrode configurations for finger movement classification using EMG. Proceedings of the 31st Annual International Conference of the IEEE $E M B S$, Minneapolis, Minnesota (pp. 2987-2990). 
Arjunan, S. P. (2008). Fractal features of surface electromyogram: A new measure for low level muscle activation. Ph.D. Dissertation, RMIT University, Melbourne, Victoria, Australia.

Arjunan, S. P., \& Kumar, D. K. (2010). Decoding subtle forearm flexions using fractal features of surface electromyogram from single and multiple sensors. Journal of NeuroEngineering and Rehabilitation, $7(53)$.

Arjunan, S. P., \& Kumar, D. K. (2011). Fractal properties of surface electromyogram for classification of low-level hand movements from single-channel forearm muscle activity. Journal of Mechanics in Medicine and Biology, 11(3), 581-590.

Barreto, A. B., Scargle, S. D., \& Adjouadi, M. (2000). A practical EMG-based human-computer interface for users with motor disabilities. Journal of Rehabilitation Research and Development, 37(1), 53-64.

Benko, H., Saponas, T. S., Morris, D., \& Tan, D. (2009). Enhancing input on and above the interactive surface with muscle sensing. Proceedings of the ACM International Conference on Interactive Tabletops and Surfaces, Banff (pp. 93-100).

Bolgla, L. A., \& Uhl, T. L. (2007). Reliability of electromyographic normalization methods for evaluating the hip musculature. Journal of Electromyography and Kinesiology, 17(1), 102-111.

Boostani, R., \& Moradi, M. H. (2003). Evaluation of the forearm EMG signal features for the control of a prosthetic hand. Physiological Measurement, 24(2), 309-319.

Boschmann, A., Kaufmann, P., Platzner, M., \& Winkler, M. (2009). Towards multi-movement hand prostheses: Combining adaptive classification with high precision sockets. Proceedings of 2nd European Conference Technically Assisted Rehabilitation, Berlin (pp. 1-4).

Brzostowski, K., \& Zieba, M. (2011). Analysis of human arm motions recognition algorithms for system to visualize virtual arm. Proceedings of the 21st International Conference on Systems Engineering, Las Vegas (pp. 422-426).

Cannan, J. A. R., \& Hu, H. (2011). Automatic circumference measurement for aiding in the estimation of maximum voluntary contraction (MVC) in EMG systems. Proceedings of the 4th international conference on Intelligent Robotics and Applications, Aachen (pp. 202-211).

Cifrek, M., Medved, V., Tonković, S., \& Ostojić, S. (2009). Surface EMG based muscle fatigue evaluation in biomechanics. Clinical Biomechanics, 24(4), 327-340.

Chan, F. H. Y., Yang, Y. S., Lam, F. K., Zhang, Y. T., \& Parker, P. A. (2000). Fuzzy EMG classification for prosthesis control. IEEE Transactions on Rehabilitation Engineering, 8(3), 305-311.

Chen, L., Geng, Y., \& Li, G. (2011). Effect of upper-limb positions on motion pattern recognition using electromyography. Proceedings of 4th International Congress on Image and Signal Processing, Shanghai (pp. 139-142).

Clancy, E. A., \& Hogan, N. (1999). Probability density of the surface electromyogram and its relation to amplitude detectors. IEEE Transactions on Biomedical Engineering, 46(6), 730-739.

De Luca, C. J. (1984). Myoelectrical manifestations of localized muscular fatigue in humans. Critical Reviews in Biomedical Engineering, 11(4), 254-279.

De Luca, C. J. (2002). Surface electromyography: Detection and recording. Retrieved from http://www.delsys.com/Attachments_pdf/WP_SEMGintro.pdf

De Luca, C. J., Gilmore, L. D., Kuznetsov, M., \& Roy, S. H. (2010). Filtering the surface EMG signal: Movement artifact and baseline noise contamination. Journal of Biomechanics, 43(8), 1573-1579.

De Leva, P. (1996). Adjustments to Zatsiorsky-Seluyanov's segment inertia parameters. Journal of Biomechanics, 29(9), 1223-1230.

Du, Y. C., Lin, C. H., Shyu, L. Y., \& Chen, T. (2010). Portable hand motion classifier for multi-channel surface electromyography recognition using gray relational analysis. Expert Systems with Applications, 37(6), 4283-4291.

Du, Y. C., Shyu, L. Y., \& Hu, W. (2006). The effect of combining stationary wavelet transform and independent component analysis in the multichannel SEMGs hand motion identification system. Journal of Medical and Biological Engineering, 26(1), 9-14. 
Esteller, R., Vachtsevanos, G., Echauz, J., \& Litt, B. (2001). A comparison of waveform fractal dimension algorithms. IEEE Transactions on Circuits and Systems I: Fundamental Theory and Applications, 48(2), 177-1831.

Fougner, A. L. (2007). Proportional myoelectric control of a multifunction upper-limb prosthesis. Master thesis, Norwegian University of Science and Technology, Norway.

Fougner, A., Scheme, E., Chan, A. D. C., Englehart, K., \& Stavdahl, Ø. (2011). Resolving the limb position effect in myoelectric pattern recognition. IEEE Transactions on Neural Systems and Rehabilitation Engineering, 19(6), 644-651.

Gitter, J. A., \& Czerniecki, M. J. (1995). Fractal analysis of the electromyographic interference pattern. Journal of Neuroscience Methods, 58(1-2), 103-108.

Gupta, V., Suryanarayanan, S., \& Reddy, N. P. (1997). Fractal analysis of surface EMG signals from the biceps. International Journal of Medical Informatics, 45(3), 185-192.

Higuchi, T. (1988). Approach to an irregular time series on the basis of the fractal theory. Physica D: Nonlinear Phenomena, 31(2), 277-283.

Hof, A. L., Pronk, C. N. A., \& van Best, J. A. (1987). Comparison between EMG to force processing and kinetic analysis for the calf muscle moment in walking and stepping, Journal of Biomechanics, 20(2), 167-178.

Hogan, N., \& Mann, R. W. (1980). Myoelectric signal processing: Optimal estimation applied to electromyography-Part I: Deviation of the optimal myoprocessor, IEEE Transactions on Biomedical Engineering, BME-27(7), 382-395.

Holzbaur, K. R., Murray, W. M., Gold, G. E., \& Delp, S. L. (2007). Upper limb muscle volumes in adult subjects. Journal of Biomechanics, 40(4), 742-749.

Hu, X., Wang, Z., \& Ren, X. (2005). Classification of surface EMG signal with fractal dimension. Journal of Zhejiang University Science B, 6(8), 844-848.

Hudgins, B., Parker, P., \& Scott, R. N. (1993). A new strategy for multifunction myoelectric control. IEEE Transactions on Biomedical Engineering, 40(1), 82-94.

Jain, S., Singhal, G., Smith, R. J., Kaliki, R., \& Thakor, N. (2012). Improving long term myoelectric decoding, using an adaptive classifier with label correction. Proceedings of the 4th IEEE RAS/EMBS International Conference on Biomedical Robotics and Biomechatronics, Roma (pp. 532-537).

Jiang, N., Muceli, S., Graimann, B., \& Farina, D. (2013). Effect of arm position on the prediction of kinematics from EMG in amputees. Medical \& Biological Engineering \& Computing, 51(1-2), 143151.

Jindapetch, N., Chewae, S., \& Phukpattaranont, P. (2012). FPGA implementations of an ADALINE adaptive filter for power-line noise cancellation in surface electromyography signals. Measurement, 45(3), 405-414.

Kamavuako, E. N., Farina, D., Yoshida, K., \& Jensen, W. (2009). Relationship between grasping force and features of single-channel intramuscular EMG signals. Journal of Neuroscience Methods, 185(1), 143-150.

Kamavuako, E. N., Rosenvang, J. C., Bøg, M. F., Smidstrup, A., Erkocevic, E., Niemeier, M. J., ... Farina, D. (2013). Influence of the feature space on the estimation of hand grasping force from intramuscular EMG. Biomedical Signal processing and Control, 8(1), 1-5.

Kaufmann, P., Englehart, L., \& Platzner, M. (2010). Fluctuating EMG signals: Investigating long-term effects of pattern matching algorithms. Proceedings of the 32nd Annual International Conference of the IEEE Engineering in Medicine and Biology Society, Buenos Aires (pp. 6357-6360).

Kendall, C., Lemaire, E. D., Losier, Y., Chan, A., \& Hudgins, B. (2012). A novel approach to surface electromyography: An exploratory study of electrode-pair selection based on signal characteristics. Journal of NeuroEngineering and Rehabilitation, 9(24).

Khushaba, R. N., \& Kodagoda, S. (2012). Electromyogram (EMG) feature reduction using mutual components analysis for multifunction prosthetic fingers control. Proceedings of the 12th International Conference on Control, Automation, Robotics and Vision, Guangzhou (pp. 1534-1539). 
Khushaba, R. N., Kodagoda, S., Liu, D., \& Dissanayake, G. (2013). Muscle computer interfaces for driver distraction reduction. Computer Methods and Programs in Biomedicine, 110(2), 137-149.

Kim, K. S., Choi, H. H., Moon, C. S., \& Mun, C. W. (2011). Comparison of k-nearest neighbor, quadratic discriminant and linear discriminant analysis in classification of electromyogram signals based on the wrist-motion directions. Current Applied Physics, 11(3), 740-745.

Kim, S. J., Jeong, E. C., Lee, S. M., \& Song, Y. R. (2012). Improvements of multi-features extraction for EMG for estimating wrist movements. The Transactions of the Korean Institute of Electrical Engineers, 61(5), 757-762.

Kundu, A. S., Mazumder, O., \& Bhaumik, S. (2011). Design of wearable, low power, single supply surface EMG extractor unit for wireless monitoring. Proceedings of the 2nd International Conference on Nanotechnology and Biosensors, Dubai (pp. 69-74).

Lawrence, J. H., \& De Luca, C. J. (1983). Myoelectric signal versus force relationship in different human muscles. Journal of Applied Physiology, 54(6), 1653-1659.

Li, K., Hewson, D. J., Duchene, J., \& Hogrel, J. -Y. (2010). Predicting maximal grip strength using hand circumference. Manual Therapy, 15(6), 579-585.

Li, G., Li, Y., Yu, L., \& Geng, Y. (2011). Conditioning and sampling issues of EMG signals in motion recognition of multifunctional myoelectric prostheses. Annals of Biomedical Engineering, 39(6), 1779-1787.

Marras, W. S., \& Davis, K. G. (2001). A non-MVC EMG normalization technique for the trunk musculature: Part 1. Method development. Journal of Electromyography and Kinesiology, 11(1), 19.

Marras, W. S., \& Sommerich, C. M. (1991). A three-dimensional motion model of loads on the lumbar spine: I. Model structure. Human Factors, 33(2), 123-137.

Merletti, R., \& Parker, P. J. (Eds.). (2004). Electromyography: Physiology, engineering, and non-invasive applications. Hoboken, NJ: John Wiley \& Sons.

Mogk, J. P. M., \& Keir, P. J. (2003). Crosstalk in surface electromyography of the proximal forearm during gripping tasks. Journal of Electromyography and Kinesiology, 13(1), 63-71.

Oatis, C. A. (2008). Kinesiology: The mechanics and pathomechanics of human movement. Philadelphia, PA: Lippincott Williams \& Wilkins.

Oskoei, M. A., \& Hu, H. (2008). Support vector machine-based classification scheme for myoelectric control applied to upper limb. IEEE Transactions on Biomedical Engineering, 55(8), 1956-1965.

Oskouei, A. H., Paulin, M. G., \& Carman, A. B. (2013). Intra-session and inter-day reliability of forearm surface EMG during varying hand grip forces. Journal of Electromyography and Kinesiology, 23(1), 216-222.

Park, S. H., \& Lee, S. P. (1998). EMG pattern recognition based on artificial intelligence techniques. IEEE Transactions on Rehabilitation Engineering, 6(4), 400-405.

Peng, C. K., Havlin, S., Stanley, H. E., \& Goldberger, A. L. (1995). Quantification of scaling exponents and crossover phenomena in nonstationary heartbeat time series. Chaos, 5(1), 82-87.

Philipson, L., \& Larsson, P. G. (1988). The electromyographic signal as a measure of muscular force: A comparison of detection and quantification techniques. Electromyography and Clinical Neurophysiology, 28(2-3), 141-150.

Phinyomark, A., Limsakul, C., \& Phukpattaranont, P. (2008). EMG feature extraction for tolerance of white Gaussian noise. Proceedings of the International Workshop and Symposium on Science and Technology, Nongkhai (pp. 178-183).

Phinyomark, A., Limsakul, C., \& Phukpattaranont, P. (2009). EMG feature extraction for tolerance of 50 $\mathrm{Hz}$ interference. Proceedings of the 4th International Conference on Engineering Technologies, Novi Sad (pp. 289-293).

Phinyomark, A., Hirunviriya, S., Phukpattaranont, P., \& Limsakul, C. (2010). Evaluation of EMG Feature Extraction for Hand Movement Recognition Based on Euclidean Distance and Standard Deviation. Proceedings of the 7th International Conference on Electrical Engineering/Electronics, Computer, Telecommunications and Information Technology, Chiang Mai (pp. 856-860). 
Phinyomark, A., Hirunviriya, S., Nuidod, A., Phukpattaranont, P., \& Limsakul, C. (2011a). Evaluation of EMG feature extraction for movement control of upper limb prostheses based on class separation index. Proceedings of the 5th Kuala Lumpur International Conference on Biomedical Engineering, Kuala Lumpur (pp. 750-754).

Phinyomark, A., Phothisonothai, M., Phukpattaranont, P., \& Limsakul, C. (2011b). Critical exponent analysis applied to surface electromyography (EMG) signals for gesture recognition. Metrology and Measurement Systems, 18(4), 645-658.

Phinyomark, A., Phukpattaranont, P., \& Limsakul, C. (2011c). Wavelet-based denoising algorithm for robust EMG pattern recognition. Fluctuation and Noise Letters, 10(2), 157-167.

Phinyomark, A., Phukpattaranont, P., Limsakul, C., \& Phothisonothai, M. (2011d). Electromyography (EMG) signal classification based on detrended fluctuation analysis. Fluctuation and Noise Letters, 10(3), 281-301.

Phinyomark, A., Chujit, G., Phukpattaranont, P., Limsakul, C., \& Hu, H. (2012a). A preliminary study assessing time-domain EMG features of classifying exercises in preventing falls in the elderly. Proceedings of the 9th International Conference on Electrical Engineering/Electronics, Computer, Telecommunications and Information Technology, Phetchaburi (pp. 1-4).

Phinyomark, A., Nuidod, A., Phukpattaranont, P., \& Limsakul, C. (2012b). Feature extraction and reduction of wavelet transform coefficients for EMG pattern classification. Elektronika ir Elektrotechnika, 122(6), 27-32.

Phinyomark, A., Phukpattaranont, P., \& Limsakul, C. (2012c). Feature reduction and selection for EMG signal classification. Expert Systems with Applications, 39(8), 7420-7431.

Phinyomark, A., Phukpattaranont, P., \& Limsakul, C. (2012d). Fractal analysis features for weak and single-channel upper-limb EMG signal. Expert Systems with Applications, 39(12), 11156-11163.

Phinyomark, A., Phukpattaranont, P., \& Limsakul, C. (2012e). Investigating long-term effects of feature extraction methods for continuous EMG pattern classification. Fluctuation and Noise Letters, 11(4), 1250028.

Phinyomark, A., Phukpattaranont, P., \& Limsakul, C. (2012f). The usefulness of wavelet transform to reduce noise in the SEMG signal. In M. Schwartz (Ed.), EMG methods for evaluating muscle and nerve function (pp. 107-132). Rijeka: InTech.

Phinyomark, A., Thongpanja, S., Hu, H., Phukpattaranont, P., \& Limsakul, C. (2012g). The usefulness of mean and median frequencies in electromyography analysis. In G. R. Naik (Ed.), Computational intelligence in electromyography analysis - A perspective on current applications and future challenges (pp. 195-220). Rijeka: InTech.

Phinyomark, A., Quaine, F., Charbonnier, S., Serviere, C., Tarpin-Bernard, F., \& Laurillau, Y. (2013a). A feasibility study on the use of anthropometric variables to make muscle-computer interface more practical. Engineering Applications of Artificial Intelligence, 26(7), 1681-1688.

Phinyomark, A., Quaine, F., Charbonnier, S., Serviere, C., Tarpin-Bernard, F., \& Laurillau, Y. (2013b). EMG Feature Evaluation for Improving Myoelectric Pattern Recognition Robustness. Expert Systems with Applications, 40(12), 4832-4840.

Phothisonothai, M., \& Nakagawa, M. (2007). Fractal-based EEG data analysis of body parts movement imagery tasks. Journal of Physiological Sciences, 57(4), 217-226.

Reaz, M. B. I., Hussain, M. S., \& Mohd-Yasin, F. (2006). Techniques of EMG signal analysis: Detection, processing, classification and applications. Biological Procedures Online, 8(1), 11-35.

Rhee, K. W., You, K. J., \& Shin, H. C. (2011). Recognition of finger motion with sEMG and gyrosensor signals. Journal of Measurement Science and Instrumentation, 2(2), 136-139.

Richman, J. S., \& Moorman, J. R. (2000). Physiological time series analysis using approximate entropy and sample entropy. American Journal of Physiology Heart and Circulatory Physiology, 278(6), H2039-H2049.

Saponas, T. S., Tan, D. S., Morris, D., \& Balakrishnan, R. (2008). Demonstrating the feasibility of using forearm electromyography for muscle-computer interfaces. Proceedings of the SIGCHI Conference on Human Factors in Computing Systems, Florence (pp. 515-524). 
Saponas, T. S., Tan, D. S., Morris, D., Balakrishnan, R., Turner, J., \& Landay, J. A. (2009). Enabling always-available input with muscle-computer interfaces. Proceedings of the ACM Symposium on User Interface Software and Technology, Victoria, BC (pp. 167-176).

Saponas, T. S., Tan, D. S., Morris, D., Turner, J., \& Landay, J. A. (2010). Making muscle-computer interfaces more practical. Proceedings of the SIGCHI Conference on Human Factors in Computing Systems, Atlanta, GA (pp. 851-854).

Sensinger, J. W., Lock, B. A., \& Kuiken, T. A. (2009). Adaptive pattern recognition of myoelectric signals: exploration of conceptual framework and practical algorithms. IEEE Transactions on Neural Systems and Rehabilitation Engineering, 17(3), 270-278.

Shenoy, P., Miller, K. J., Crawford, B., \& Rao, R. P. N. (2008). Online electromyographic control of a robotic prosthesis. IEEE Transactions on Biomedical Engineering, 55(3), 1128-1135.

Shyu, L. Y., Chen, J. Y., Tatn, R. W., \& Hu, W. (2002). A new electrode system for hand action discrimination. Journal of Medical and Biological Engineering, 22(4), 211-217.

Smith, R. J., Huberdeau, D., Tenore, F., \& Thakor, N. V. (2009). Real-time myoelectric decoding of individual finger movements for a virtual target task. Proceedings of the 31 st Annual International Conference of the IEEE EMBS, Minneapolis, Minnesota (pp. 2376-2379).

Smith, R. J., Tenore, F., Huberdeau, D., Etienne-Cummings, R., \& Thakor, N. V. (2008). Continuous decoding of finger position from surface EMG signals for the control of powered prostheses. Proceedings of the 30th Annual International Conference of the IEEE EMBS, Vancouver, British Columbia (pp. 197-200).

Subasi, A. (2012). Medical decision support system for diagnosis for neuromuscular disorders using DWT and fuzzy support vector machines. Computers in Biology and Medicine, 42(8), 806-815.

Subasi, A. (2013). Classification of EMG signals using PSO optimized SVM for diagnosis of neuromuscular disorders. Computers in Biology and Medicine, 43(5), 576-586.

Tang, X., Liu, Y., Lv, C., \& Sun, D. (2012). Hand motion classification using a multi-channel surface electromyography sensor. Sensors, 12(2), 1130-1147.

Taylor, R. (1990). Interpretation of the correlation coefficient: A basic review. Journal of Diagnostic Medical Sonography, 6(1), 35-39.

Tenore, F. V. G., Ramos, A., Fahmy, A., Acharya, S., Etienne-Cummings, R., \& Thakor, N. V. (2007). Towards the control of individual fingers of a prosthetic hand using surface EMG signals. Proceedings of the 29th Annual International Conference of the IEEE EMBS, Lyon (pp. 6145-6148).

Tenore, F. V. G., Ramos, A., Fahmy, A., Acharya, S., Etienne-Cummings, R., \& Thakor, N. V. (2009). Decoding of individual finger movements using surface electromyography. IEEE Transactions on Biomedical Engineering, 56(5), 1427-1434.

Thongpanja, S., Phinyomark, A., Phukpattaranont, P., \& Limsakul, C. (2013). Mean and median frequency of EMG signal to determine muscle force based on time-dependent power spectrum. Elektronika ir Elektrotechnika, 19(3), 51-56.

Tkach, D., Huang, H., \& Kuiken, T. A. (2010). Study of stability of time-domain features for electromyographic pattern recognition. Journal of NeuroEngineering and Rehabilitation, 7(21).

Vera-Garcia, F. J., Moreside, J. M., \& McGill, S. M. (2010). MVC techniques to normalize trunk muscle EMG in healthy women. Journal of Electromyography and Kinesiology, 20(1), 10-16.

Vigreux, B., Cnockaert, J. C., \& Pertuzon, E. (1979). Factors influencing quantified surface EMGs. European Journal of Applied Physiology and Occupational Physiology, 41(2), 119-129.

Winter, D. A. (1990). Biomechanics and Motor Control of Human Movement. New York, Wiley \& Sons.

Wei, L., Hu, H., \& Zhang, Y. (2011). Fusing EMG and visual data for hands-free control of an intelligent wheelchair. International Journal of Humanoid Robotics, 8(4), 707-724.

Woods, J. J., \& Bigland-Ritchie, B. (1983). Linear and non-linear surface EMG/force relationships in human muscles. An anatomical/functional argument for the existence of both. American Journal of Physical Medicine, 62(6), 287-299. 
Yang, S. W., Lin, C. S., Lin, S. K., \& Lee, C. H. (in press). Design of virtual keyboard using blink control method for the severely disabled. Computer Methods and Programs in Biomedicine, doi:http://dx. doi.org/10.1016/j.cmpb.2013.04.012.

You, K. J., Rhee, K. W., \& Shin, H. C. (2011). Finger flexion motion inference from sEMG signals. Journal of Measurement Science and Instrumentation, 2(2), 140-143.

You, K. J., Rhee, K. W., \& Shin, H. C. (2010). Finger motion decoding using EMG signals corresponding various arm postures. Experimental Neurobiology, 19(1), 54-61.

Young, A. J., Hargrove, L. J., \& Kuiken, T. A. (2011). The effects of electrode size and orientation on the sensitivity of myoelectric pattern recognition systems to electrode shift. IEEE Transactions on Biomedical Engineering, 58(9), 2537-2544.

Young, A. J., Hargrove, L. J., \& Kuiken, T. A. (2012). Improving myoelectric pattern recognition robustness to electrode shift by changing interelectrode distance and electrode configuration. IEEE Transactions on Biomedical Engineering, 59(3), 645-652.

$\mathrm{Yu}$, S., Jeong, E., Hong, K., \& Lee, S. (2012). Classification of nine directions using the maximum likelihood estimation based on electromyogram of both forearms. Biomedical Engineering Letters, 2(2), 129-137.

Zardoshti-Kermani, M., Wheeler, B. C., Badie, K., \& Hashemi, R. M. (1995). EMG feature evaluation for movement control of upper extremity prostheses. IEEE Transactions on Rehabilitation Engineering, 3(4), 324-333.

Zhang, X., Chen, X., Zhao, Z. Y., Li, Q., Yang, J. H., Lantz, V., \& Wang, K.Q. (2008). An adaptive feature extractor for gesture SEMG recognition. Proceedings of the 1st International Conference on Medical Biometrics, Hong Kong (pp. 83-90).

Zhang, X., \& Zhou, P. (2012). Sample entropy analysis of surface EMG for improved muscle activity onset detection against spurious background spikes. Journal of Electromyography and Kinesiology, 22(6), 901-907.

Zhao, J., Jiang, L., Cai, H., Liu, H., \& Hirzinger, G. (2006a). A novel EMG motion pattern classifier based on wavelet transform and nonlinearity analysis method. Proceedings of IEEE International Conference on Robotics and Biomimetics, Kunming (pp. 1494-1499).

Zhao, J., Xie, Z., Jiang, L., Cai, H., Liu, H., \& Hirzinger, G. (2006b). EMG control for a five-fingered underactuated prosthetic hand based on wavelet transform and sample entropy. Proceedings of IEEE/RSJ International Conference on Intelligent Robots and Systems, Beijing (pp. 3215-3220).

\section{ADDITIONAL READING SECTION}

\section{A review of EMG signal processing and pattern recognition techniques}

Ahsan, Md. R., Ibrahimy, M. I., \& Khalifa, O. O. (2009). EMG signal classification for human computer interaction: A review. European Journal of Scientific Research, 33(3), 480-501.

Franti, E., Milea, L., Butu, V., Cismas, S., Lungu, M., Schiopu, P., Barbilian, A., Plavitu, A. (2012). Methods of acquisition and signal processing for myoelectric control of artificial arms. Romanian Journal of Information Science and Technology, 15(2), 91-105.

Herle, S., \& Man, S. (2009). Processing surface electromyographical signals for myoelectric control. In T. Y. Kheng (Ed.), Rehabilitation engineering (pp. 223-244). Rijeka: InTech.

Li, G. (2011). Electromyography pattern-recognition-based control of powered multifunctional upperlimb prostheses. In J. Mizrahi (Ed.), Advances in applied electromyography (pp. 99-116). Rijeka: InTech.

Micera, S., Carpaneto, J., \& Raspopovic, S. (2010). Control of hand prostheses using peripheral information. IEEE Reviews in Biomedical Engineering, 3, 48-68.

Oskoei, M. A., \& Hu, H. (2007). Myoelectric control systems - A survey. Biomedical Signal Processing and Control, 2(4), 275-294. 
Peerdeman, B., Boere, D., Witteveen, H., in 't Veld, R. H., Hermens, H., Stramigioli, S., ... Misra, S. (2011). Myoelectric forearm prostheses: State of the art from a user-centered perspective. Journal of Rehabilitation Research \& Development, 48(6), 719-738.

Phinyomark, A., Phukpattaranont, P., \& Limsakul, C. (2011). A review of control methods for electric power wheelchairs based on electromyography (EMG) signals with special emphasis on pattern recognition. IETE Technical Review, 25(4), 316-326.

Zecca, M., Micera, S., Carrozza, M. C., \& Dario, P. (2002). Control of multifunctional prosthetic hands by processing the electromyograpic signal. Critical Reviews in Biomedical Engineering, 30(4-6), 459-485.

\section{KEY TERMS AND DEFINITIONS}

Keyword: Anthropometry, Bi-deltoid Breadth, Electromyography (EMG) Signal, Feature Extraction, Forearm Circumference, Multifunction Myoelectric Control, Muscle-Computer Interface, Gesture Recognition.

- Anthropometry is a measurement of the dimensions of the different parts of body.

- Bi-deltoid Breadth (shoulder breadth) is measured from the maximum horizontal breadth across the body at the level of the deltoid landmarks.

- Electromyography (EMG) Signal is an electrophysiological signal which is measured from the proposed muscle by electrodes during movements.

- Feature Extraction is a technique to transform original signal into a reduced set of features, which highlight the relevant structures and discard the irrelevant parts in the original signal.

- Forearm Circumference is the linear distance around the forearm, which is usually measured at the level of maximum forearm circumference.

- Multifunction Myoelectric Control is an advanced technique concerned with the preprocessing, feature extraction, dimensionality reduction, and pattern classification of myoelectric (or EMG) signals to control external devices with many functions.

- Muscle-Computer Interface is an interface between users based on EMG signals via muscles and computers.

- Gesture Recognition is a technique to interpret and classify the human gestures via mathematical algorithms. 